\title{
ESTADO ACTUAL DEL CARCINOMA DE CUELLO UTERINO*
}

\author{
Doctor Gilberto Angel M. **
}

\section{Introducción}

En la primera semana de febrero de I964, se verificó en México la Convención Internacional contra el cáncer y la Reunión Latinoamericana de Cancerología. Simultáneamente, durante 6 días se trataron temas relacionados con el cáncer del pene, carcinoma de endometrio y carcinoma cervical; el carcinoma del cervix fue debatido ampliamente en todos sus aspectos por expositores, en su gran mayoría, de reconocido prestigio internacional y gran experiencia. Nos ha parecido de interés divulgar entre los colegas interesados en estos temas, el concepto mundial actual del carcinoma del cuello, pues si tenemos en cuenta los países participantes y la calidad de sus representantes, el concepto global del tema que nos ocupa representa los conocimientos mundiales que en la actualidad se tienen del carcinoma del cuello uterino. Queremos expresar nuestros sinceros agradecimientos a la doctora Nubia Muñoz y al doctor Pelayo Correa, también presentes en este certamen, por las informaciones suministradas de las sesiones, que por ser en forma simultánea, no tuve la oportunidad de asistir.

\section{Participantes}

23I delegados asistieron de $3^{8}$ países en la siguiente proporción: Alemania I, Africa del Sur 2, Argent1na 22, Australia 2, Bélgica I, Bolivia 2, Brasil 5. Costa Rica I, Canadá 4, Colombia 6, Cuba I, Chile 4, Ecuador I, El Salvador I, España I, Estados Unidos 40, Filipinas I, Francia 4, Gran Bretaña 4, Ghana I, Grecia 2, Guatemala I, Honduras I, India 2, Italia 2, Jamaica 2, Japón I, Nicaragua I, México I04, Paraguay I, Perú 2, Singapur I, Suecia I, Uganda I, Rusia 3, Uruguay I, Venezuela 6.

\footnotetext{
* Resumen de la Convención Internacional contra el Cáncer y Reunión Latinoamericana de Cancerologia, reunida en México en febrero de 1964.

* Del Departamento de Morfología de la Facultad de Medicina, Universidad del Valle.
} 


\section{Epidemiología}

De la Patología geográfica recopilada en Estados Unidos y Europa Occidental, el doctor John C. Bailer deduce que la frecuencia del cáncer cervical es mayor en mujeres con muchos hijos. Existe mayor incidencia en los grupos con pocas posibilidades económicas y es más frecuente en la población urbana que rural. El doctor Richard Doll $\left({ }^{11}\right)$ encuentra casi siempre una relación inversamente proporcional al carcinoma de mama. En el Japón, Dinamarca y Estados Unidos encuentra un factor genético importante, siendo más frecuente en las mujeres del grupo sanguíneo A y una frecuencia mayor en las personas con deficiencia vitamínica $\mathrm{A}$, recordando la importancia dicha vitamina en la constitución de los epitelios. Encuentra una mayor incidencia en las mujeres fértiles, relacionando su frecuencia a la edad del primer coito. Analiza una relación con el orgasmo en la mujer, siendo más frecuente en las mujeres sexualmente apáticas. El parto por si mismo, no lo considera importante como factor desencadenante, radicando su importancia en el coito y recalca la baja incidencia en las judías, donde existe un alto número de circuncidados masculinos.

\section{Incidencia}

En el Brasil entre 202.447 personas examinadas se encuentran $15.049 \mathrm{ca}$ sos de carcinomas y de ellos 2.262 fueron de cáncer uterino. La frecuencia relativa del carcinoma del útero con relación al cáncer en general, fue del $14 \%$. El predominio del Ca. cervical sobre el del cuerpo fue del $88 \%$. El carcinoma in situ, tuvo una frecuencia del 3,9\%. En Jamaica, el carcinoma del cervix, representó el $30 \%$ de los tumores malignos femeninos. En Colombia, el $87.5 \%$ de los procesos malignos de la mujer, corresponden al útero. En El Salvador el $89 \%$ de los tumores malignos femeninos, corresponden a Ca. del cuello. En Estados Unidos la incidencia es igualmente alta, pero cada año se encuentran menor número de carcinomas cervicales avanzados, aumentando la cifra de diagnósticos precoces y curaciones totales o relativas, por la gran campaña verificada con el $\mathrm{Pa}$ panicolaou y el elevado número de diagnósticos precoces, inducidos por la citología.

\section{Edad}

Todos los estudios estadísticos coinciden en que la edad predilecta para el Ca. de cuello, es entre los 40 y 50 años. En El Salvador su mayor frecuencia se encuentra entre los 35 y 44 años.

Inducción experimental del cáncer uterino en animales de laboratorio

El doctor Gardner ('"丷) encuentra que el carcinoma cérvico-uterino ocurre raras veces en ratas y conejas. Los del cuerpo uterino ocurren más frecuentemente en estas últimas, en combinación con hipertrofia ovárica, adrenocortical e hipofisiaria. En las 
conejas, se observa primero hiperplasia uterina, adenomatosa y finalmente adenocarcinomas. Altas dosis de estrógenos preparan el terreno si no existe dosis de progesterona que contrarresten su acción. Las conejas, sólo producen progesterona con el coito y las vírgenes viven en un continuo hiper-estrogenismo. Los cánceres cérvico-uterinos han sido inducidos en ratas por inyecciones prolongadas de hormonas estrógenas y propionato de testosterona combinados. Hay un terreno evidente receptivo, pues hay familias de ratas más predispuestas que otras al Ca. La aplicación tópica de estrógenos induce al Ca. cérvico-uterino y demuestra acción carcinogénica de los estrógenos. Hidrocarbonos carcinogénicos aplicados al cuello y vagina de las ratas, durante períodos prolongados, inducen carcinoma uterino y cérvico uterino; no han sido observados en monas, a las que se les ha suministrado estrógenos durante períodos prolongados. Cotchin ${ }^{6}$ ) encuentra que el carcinoma del cervix es exclusivo de la mujer y experimentalmente se logra producir adenocarcinomas endometriales a vacas y conejas.

\section{Aspectos endocrinológicos}

El doctor Iglesias $\left({ }^{18}\right)$ expone que bajo el estímulo estrogénico, en ausencia de progesterona, la célula endometrial no alcanza su diferenciación completa, quedándose inmadura en condiciones de seguir multiplicándose. La hiperplasia y malignidad, de- ben considerarse como resultado de una frustración de la célula endometrial, estimulada por estrógenos, que espera la acción de la progesterona. Sc hacen comentarios muy favorables, en el tratamiento del adenocarcinoma de endometrio con progesterona y sus derivados.

El doctor Gregorio Pincus $\left({ }^{23}\right)$ presenta un estudio estadístico sobre la frecuencia de citologías sospechosas y positivas (Papanicolaou tipos III a V) en mujeres de seis localidades. En la zona del Caribe de cada r.ooo mujeres 50 representan este tipo de citologías y usan contraceptivos, 54 artefactos intra-uterinos y 26 usan como anticoncepcional Enovid. Siendo el tipo de citologías positivas menos frecuentes entre las que usan contraceptivos esteroides y más frecuente entre las que usan otros métodos, sugiere la posibilidad de que estos esteroides puedan tener un efecto preventivo en el Ca. del útero.

\section{Herencia}

El doctor Baruchi Blumberg $\left({ }^{4}\right)$ afirma que no existen en la actualidad factores genéticos determinados. Estudios familiares y gemelares, indican que es muy poco probable que el Ca. del cuello se herede por simples leyes Mendelianas. Persiste la posibilidad de que la herencia a la susceptibilidad y los factores de resistencia que intervienen con los agentes exógenos, puedan ser responsables en la incidencia variable, que se encuentra en diferentes áreas geográficas. 


\section{Etiología}

Se ha estudiado desde hace 120 años $\left({ }^{11}\right)$ y no existe una causa única, específica y determinada. Indudablemente existen multitud de factores que en mayor o menor grado hacen vulnerable el epitelio cervical, jugando un papel muy importante el terreno $\left({ }^{42}\right)$ y condiciones propias de la paciente. La sífilis parece jugar un factor muy importante como causa adyuvante. Teda causa que obre como factor irritativo, como muchos partos, inflamaciones, erosiones evolutivas, anticoncepcionales caústicos, etc. contribuyen en mayor o menor grado, según la suceptibilidad y terreno de la paciente, a desarrollarlo. Las estadísticas recopiladas, relacionadas con edad del primer coito, frecuencia de éste, ausencia total del carcinoma del cuello en las vírgenes y mayor incidencia en mujeres que tienien la oportunidad de practicar el coito con diferentes hombres, inducen a pensar, considerando la poca frecuencia del Ca. del cervix en la población israelita, que en el coito radica una de las mayores causas, muy posiblemente atribuída al esmegma prepucial. Es posible que en su constitución química jueguen factores cancerígenos, diferentes al que se encuentra en el esmegma de la mujer, pues bien se sabe que el carcinoma del clítoris es sumamente raro. Deben existir substancias diferentes para cada hombre, como agentes cancerígenos. En los nativos y poblaciones donde la higiene tanto masculina como femenina es muy precaria, la incidencia es mayor. Se insiste mucho en la investiga- ción del factor higiene, que parece ocupar en la etiología del carcinoma cervical, el primer puesto. Se le atribuye al coito un factor muy importante en la etilogía del Ca. cervical, argumentando que es desconocido en las vírgenes, espontáneamente ningún animal lo presenta y desde el punto de vista biológico, el hombre es el animal que más contactos sexuales tiene. En las otras especies, sólo se verifica en la época de celo y la cantidad total de coitos que tiene la hembra animal durante su vida, es mínimo comparado con el hombre.

\section{Diagnóstico}

La citología está reconocida por todos, como el primer paso útil y efectivo en el diagnóstico precoz. La única manera de comprobarlo, es por medio de la biopsia bien tomada y del sitio electivo. La Colposcopia es para el doctor Joao Paulo Rieper $\left({ }^{+1}\right)$ el mejor sistema que permite tomar una biopsia, sin peligro de tomar los sitios sanos. Afirma que se usa desde hace 40 años en Alemania, Suiza, Francia, Italia, Hungría, Checoeslovaquia, Rusia, Egipto, Brasil, Uruguay. Argentina, pero que en Estados Unidos ha faltado el ginecólogo de suficiente prestigio, que sea capaz de enseñarla, imponerla y aprovechar de ella sus magníficos resultados. Entre $\mathbf{1 5 . 0 0 0}$ colposcopias realizadas en el Brasil, se descubrieron 843 estados pre-malignos y 9I carcinomas in situ. La colposcopia sola indujo al diagnóstico correcto en el $97 \%$ de los casos. Combinada con la citología, descubre el carcinoma en sus primeros pasos en 
el $98 \%$ de los casos. Anota como desventajas de la colposcopia y colpomicroscopia, que es un elemento costoso, difícil de interpretar, por lo que se requiere mucho entrenamiento y conocimiento histológico y patológico del cervix. Para el doctor T. Antoine $\left({ }^{1}\right)$, el diagnóstico precoz se inicia con un examen a conciencia por parte del médico general o ginecólogo. Es frecuente ver en todas partes del mundo, que la falla en el diagnóstico precoz no está en la paciente sino en el médico, por falta de un buen examen. Insiste en la importancia del test de Schiller, al alcance de todos y tratar de aprovechar, siempre que sea posible, la valiosa ayuda de la citología, la colposcopia y la colpomicroscopia.

\section{Carcinoma in situ}

Los cambios atípicos limitados al epitelio ectocervical, con conservación de la capa basal y ausencia total de invasión, es para algunos alemanes, lesión diferente al carcinoma in situ, pues no todos pasan a su carcinoma invasivo. Durante ro años dejan 127 mujeres consideradas como en el "borderline" y de éstas 34 desarrollaron un carcinoma invasivo $(27 \%)$, 50 permanecieron estacionarias, 30 desaparecieron y 13 pacientes se perdieron de vista. No parece ser la conducta ideal, pues no sabemos cuál va a ser invasor o no. No todos los carcinomas invasivos, se puede afirmar, que pasen siempre por la etapa del in situ, ni todos los in situ los podemos controlar hasta el preciso momento que inician su invasión. Todo Ca. in situ se debe considerar como un carcinoma potencial, porque no sabemos todavía, cuáles factores van a obrar en favor o en contra. El doctor Taylor $\left({ }^{45}\right)$ opina que no existe ninguna evidencia que nos permita afirmar que una displasia activa o carcinoma in situ, termine en curación o en $\mathrm{Ca}$. invasivo. El peligro de dejar los Ca. in situ en observación, está respaldado con 327 casos de carcinoma in situ, tratados con histerectomía total, donde se observaron 6 casos de recurrencia y si esto prueba lo poco que savemos acerca del carcinoma in situ, no existe razón para esperar su invasión y comprobar su diagnóstico. La mejor terapia y de mejores resultados, es la histerectomía en las mujeres que no esperan tener más familia y la conización muy controlada en pacientes jóvenes con deseos de iniciar o aumentar su descendencia. La conización bien ejecutada, no ha impedido a muchas mujeres, tener hijos por vías naturales. La cirugía, bien sea expresada en la histerectomía o conización, es la única que está autorizado el médico hoy en día a seguir, pues la radioterapia en este tipo de lesión, es actualmente muy incierta y con estadísticas muy contradictorias. Koss $\left({ }^{20}\right)$ dice que en el Ca. in situ existen anormalidades en los cromosomos; son diploides y en el invasivo tetraploides, por lo que no responden a la radioterapia externa.

\section{Radioterapia y cirugía}

En el trabajo póstumo presentado del doctor Joe V. Meigs * $\left({ }^{24}\right)$ termina después de muchas estadísticas y con- 
sideraciones, diciendo que el carcinoma del útero en general, "siempre se debe extirpar quirúrgicamente, desde que las condiciones lo permitan" haciendo los tratamientos pre o postoperatorios según las circunstancias. El doctor Kottmaier de Suecia $\left({ }^{19}\right)$ opina que el tratamiento radioterápico y sus resultados no son fáciles de generalizar, pues dependen mucho de la manera de reaccionar la paciente a los diferentes métodos y a la técnica usada. De 3.484 pacientes tratados con rayos $\mathrm{x}$ y radium combinados, se obtiene una supervivencia a los 5 años del $76,9 \%$ en pacientes del estado I, 53,7\% de pacientes del estado II. El doctor Paterson $\left({ }^{3 *}\right)$ anota que a los 5 años pacientes del estado III tratadas solamente con radium, sobreviven en el $59 \%$ y la combinación del radium y rayos $\mathrm{x}$ le dio una supervivencia del $62 \%$. Ha usado desde el año de 1955 la tele-cobalto-terapia en cerca de 100.000 pacientes $y$ aprecia una mejor tolerancia, con reacciones de poca importancia. En el estado II es muy buena, pero no modifica mucho los ganglios cuando tienen metástasis. La telecobalto sola no es buena, pero da magníficos resultados combinándola con radium o rayos $\mathrm{x}$. La combinación de radium y rayos $\mathrm{x}$, le dió una supervivencia a los 5 años, en pacientes del estado III del $35 \%$ y en cambio la combinación de radium y telecobalto subió al $43 \%$. Secuelas graves por irradiación, se observaron en un $1,9 \%$, con una proporción más o menos similar a la que se puede obtener con la cirugía. El opina que siempre se debe radiar en los casos III y IV, hasta que se demuestre que existe otro tratamiento mejor.

El doctor Alexander Brunschwig(") observa un grupo de 1.778 mujeres tratadas por carcinoma de cuello, la mayoría por irradiación y anota una recurrencia del $47 \%$. Fueron tratadas nuevamente por cirugía, con una supervivencia a los 5 años del $25 \%$. Analiza muchos factores en pro y en contra de cada uno de los sistemas y afirma que en el momento actual en los carcinomas del estado II en adelante, el mejor tratamiento es la cirugía cuando esta es posible, con irradiación posterior, dependiendo el éxito en gran parte de la preparación y eficiencia, tanto del cirujano como del radioterapeuta, factor que no siempre se tiene en cuenta, al presentar las estadísticas. El doctor M. Lalane de Francia $\left({ }^{21}\right)$ opina que el telecobalto es el mejor sistema, quema menos, da menores secuelas y esteriliza más.

\section{Quimioterapia en cáncer cuello uterino}

El doctor Richard R. Trussell $\left({ }^{+6}\right)$ presentó una estadística realizada en Uganda, donde las condiciones quirúrgicas son muy precarias y la radioterapia no existe. Afirma que hoy en día la quimioterapia tiene poco que ofrecer en Ca. del cervix, pero la situación cambinará muy posiblemente, cuando se seleccionen mejor las drogas, se combine con otros tratamientos y en pacientes seleccionados. La 
terapéutica intra-arterial ha regresado algunos Ca. avanzados del cérvix, pero en forma incompleta y no persistentes. Es más efectiva, en pacientes que no han sido sometidos a procedimientos quirúrgicos extensos o a radioterapia intensa, pero no debe sustituír primariamente a los métodos que ya han probado su eficacia. El uso de la quimioterapia intra-arterial, puede ser motivo de ensayo cuando los métodos clásicos han fallado o no son aplicables.

\section{Conclusiones}

Globalmente se pueden reducir a los siguientes puntos:

La causa inmediata es desconocida pero depende ampliamente, de factores extrínsecos siendo los más importantes los siguientes:

La enfermedad es prácticamente desconocida en mujeres sin experiencia sexual y es generalmente más frecuente entre las casadas que en las solteras. Tiene mayor incidencia en las que inician relaciones sexuales más temprano. Es una entidad rara en comunidades judías y en las que los hombres son circuncidados. Tiene mayor incidencia entre las mujeres que han tenido muchos embarazos, aunque no está bien claro si la mul- tiparidad juega un papel adicional al del coito. La edad predilecta fluctúa, en la mayor parte de los países, entre los 40 y 50 años. Es difícil de obtener en los animales de experimentación, siendo los más susceptibles la coneja y la rata. Es más frecuente en personas del grupo sanguíneo A y cuando hay carencia de Vitamina A. Los esteroides parecen obrar favorablemente en este tipo de enfermedad. No parece probable que la herencia juegue un papel directo, pero sí el terreno. La citología sigue siendo el mejor sistema para detectar el carcinoma en sus primeros estados, no olvidando la importancia del buen examen clínico. La colpocitología y colpomicroscopía, son ayuda muy valiosa en su diagnóstico precoz. El carcinoma in situ se debe considerar como potencialmente invasor y su tratamiento quirúrgico, según los casos, está indicado, y no la radioterapia, por sus anormalidades cromosómicas que parece lo hacen refractario a este tipo de tratamiento. El carcinoma uterino, siempre que sea posible, se debe extirpar, no olvidando los benéficos resultados que brinda el radium, rayos $\mathrm{x}$ y cobaltoterapia. La quimioterapia, no ha demostrado hasta el presente los resultados que se esperaban y hoy en día ofrece muy pocos beneficios al $\mathrm{Ca}$. del útero. 


\section{TRABAJOS PRESENTADOS}

1. ANTOINE T. I., Universitats-Frauenklinik, Viena, Austria: Aspectos clínicos en el diagnóstico del cáncer cervical.

2. BOYES D. A., The British Columbia Cancer Institute, Vancouver, B. C.: Programa general de trabajos en cancer cervical.

3. BRUNSCHWIG ALEXANDER. Memorial Hospital, New York: El problema quirúrgico del cáncer cervical.

4. BLUMBERG BARUCH S., Estados Unidos: Factores hereditarios en relación con eI cáncer del útero.

5. CAMPOS JORGE, Lima, Perú: Naturaleza y diagnóstico del carcinoma in situ.

6. COTCHIN E., Inglaterra: Cáncer uterino espontáneo en animales.

7. CORREA FElayo, Cali, Colombia: Patología de los tumores malignos dei útero.

8. COSTA POLUIS, Buenos Aires, Argentina: Tratamiento por radiaciones del cáncer del pene.

9. DAVIS HUGH, John Hopkins University, Baltimore, Maryland, Estados Unidos: EI uso de especímenes citológicos para el control del cáncer.

10. DAvalos E., México: Antropología en relación al cáncer.

11. DOLL RICHARD, Inglaterra: Etiología del cáncer del cérvix.

12 GARDNER W. V., Estados Unidos: Introducción experimental de cáncer uterino en animales de laboratorio.

13. GRAHAM JULIO C., Instituto Nacional de México: El especimen de biopsia cervical.

14. GENTIL FERNANDO, Instituto Central del Cáncer, Sao Paolo, Brasil: Manejo total del cáncer de pene.

15. GARCiA GERMAN, Sanatorio español, México: Epidemiología del carcinoma cérvicouterino en México.

16. HAAM EMMERICH, Estados Unidos: Histoquímiea y la ultraestructura de los tumores del útero.

17. HADDOW A., Presidente de la Unión Internacional contra el Cáncer: Carcinogenéti$\cos y$ sus relaciones con la terapéutica química y biológica.

18. IGlestas RIgOBERTO, Chile: Aspectos endocrinológicos del cáncer del útero.

20. KOSS LeOPOLDO G., Memorial Hospital, New York: Diagnóstico citológico del carcinoma del cérvix.

21. LALANNE M., Institut Gustave Rous, Villejuif, France: Radioterapia de supervoltaje en el carcinoma cervical.

22. MORICARD R.: Faris, Francia: Metabolisme de la $3 \mathbf{H}$ Thymidine in the uterus with reference to cancer and pre-cancerous lesions.

23. MARTINS FRANCIA, Sao Paulo, Brasil: Radioterapía y cirugía combinada en carcinoma cervical.

24. MEIGS V. JOE, Bostion Mass, Estados Unidos (Lectura): Tratamiento quirúrgico del cáncer del cérvix.

25. MARTINEZ RAFAEL, México: Resultados preliminares en los programas del Seguro Social en la detención del cáncer cervical.

26. OCHOA SEVERO, Premio Nobel de Medicina: La clave genética, base química de la herencia. 
27. MONTAÑO GUILLERMO, México: Hechos experimentales endocrinos en tratamiento del cáncer del cérvix.

28. MUrray J. F., Johannesburg, Africa del Sur: Cáncer del pene en Africa del Sur.

29. OOTA KUNIO, Instituto del Cáncer, Toshimu-ku-, Japón: Cáncer del pene en Japón.

30. PRUDENTE ANTONIO, Río de Janeiro, Brasil: Programas en comunidad para controlar el cáncer del cérvix.

31. PAVlosviky ALEJANDRO, Buenos Aires: Papanicolaou, microscopia de fase $y$ electrónica en cáncer cervical.

32. PINCUS GREGORY, The Worcester Foundation, Shrewsbury, Mass., Estados Unidos: El potencial de manipulación de hormonas en la prevención del cáncer del cérvix.

33. PATERSON RALSTON, Manchester, Inglaterra: Pruebas clínicas del cáncer del cérvix.

34. LAgUNA JULIETA, México: Citologia exfoliativa en la patología geográíca del cáncer.

35. PACK GEORGE T.: Filosofía de la terapéutica anticancerosa y futuro de la cancerología.

36. FAYMASTER JAL, Bombay, India: Cáncer del pene.

37. RIVEROS MANUEL, Asunción, Paraguay: Tratamiento quirúrgico radical del cáncer del pene.

38. RAVENTOS ENRIQUE, Santiago de Chile: El papel de la radioterapia en el cáncer del pene.

39. RAMOS JUAN-VALENCIA, Venezuela: Tratamiento del cáncer del pene.

40. READ CLIFTON R., American Cancer Society, New York: Educación públiea en citología para cáneer cervieal en Estados Unidos.

41. RIEPER PAULO JOE, Río de Janeiro, Brasil: Colposcopia y Colpomicroscopia del cáncer cervical.

42. SAMMARTINO RODOLFO, Buenos Aires: Discusión sobre lesiones asociadas del útero, y órganos pélvicos de posible significado etiobógico en cáncer del cérvix y cuerpo

43. SIRTORI CARLO, Milán, Italia: Hechos anatomopatológicos en cáncer del cuello uterino.

44. TERzANO GUtllermo, Buenos Aires: Entrenamiento de personal profesional y técnico en citología.

45. TAYLOR HOWARD, Universidad de Columbia, Ney York, N. Y.: Manejo del carcinoma in situ del cérvix.

46. TRUSSELL RICHARD, Makeve College Medical School, Kampala, Uganda, Africa: Quimioterapia en cáncer del cuello uterino.

47. ZUCKERMANN CONRADO, Instituto Nacional de Cancerología, México: Experiencia en el tratamiento del cáncer del cuello uterino.

48. Distribución geográfica del carcinoma del útero en los siguientes países: Argentina doctor Abel Canónigo; Brasil, doctor Manuel Barreto; Chile, doctor Juan Moroder; Colombia, doctor Augusto Buendia; Ecuador, doctor M. Hinojosa; México, doctor R. Contreras; Paraguay, doctor Pedro Rolón; Uruguay, doctor M. Narancio; Bolivia, doctor N. Machicado; Venezuela, doctor Edgar Grossman; Perú, doctor E. G. Cáceres; Centro-américa, doctor Narciso Díaz; Zona del Caribe, doctor Donald Watler; En el Africa, doctor John Higginson; Europa, Norteamérica, Medio Oriente y Asia, doctor John Bailer. 